\title{
UNA FUNCIÓN DE PRODUCCIÓN AGREGADA, SU DESCOMPOSICIÓN SECTORIAL Y UNA DEMANDA DERIVADA DE FACTORES EN UNA ECONOMÍA ABIERTA: EL CASO DE MÉXICO Joaquín Tapia Maruri*
}

\author{
Analista Económico
}

(Recibido 11 de mayo 2005, aceptado 16 de agosto 2005)

\section{Resumen}

De acuerdo a la función de producción agregada de la economía, esta podría considerarse débilmente separable. No obstante, es posible que esta, se desagregue en sus componentes de demanda; en un enfoque por el lado de producción, a saber consumo, inversión, exportaciones e importaciones. Desde luego, que al establecerse estas relaciones, se pueden evaluar las posibilidades de sustitución o complementariedad entre importaciones e inversión o entre exportaciones y consumo o inversión etc. Dado que la función de producción, es derivada de un conjunto o set de tecnología, en base a la utilización de los factores productivos, se puede analizar también la intensidad de uso de los factores en la producción de este tipo de bienes. Es decir, si para producir bienes de inversión o exportaciones, se requiere una cantidad mayor de bienes importados; o bien un cambio en los precios relativos, como el tipo de cambio real o los precios de los bienes comerciables internacionalmente, a que sectores productivos afectarán más.

\section{Abstract}

According to the aggregated production function in the economy, it could be considered as a weakly separable. Even though, it is possible to disaggregated in it components such as consumption, investment, exports and imports. Of course, that this behavioral relations could be evaluated to see a degree of substitution or complementarities between imports and investment or among exports and consumption or investment, etc. Given the fact, that the production function is derivated from a technology set, it could be stated the intensity use factor for the aggregated production function as well as the components such as consumption, investment, exports and imports. As a result, it could be address too, that for the investment or export functions it is required a high imports quantities, or for a given change in the vector relative prices, such as real exchange rate, or prices for tradable goods, which productive sectors will be more damaged.

\section{Clasificación JEL: D24, D51, D60}

Palabras clave: Función de producción, Efectos sustitución y complementarios, Demanda derivada de factores y por sectores

* Cerro de la libertad No. 439, Col. Campestre Churubusco, Coyoacán, c.p. 04200, México, D. F. Teléfono: +52(55) 5544-2685. Correo Electrónico: jtapiamaruri@yahoo.com

A la memória de Jorge Arrieta Nieto. Agradezco a Ulrich Kolih sus puntos de vista y observaciones, así como a Javier Salas Martín del Campo y Víctor Guerrero por sus comentarios y a 2 árbitros anónimos. 


\section{Introducción}

La teoría del crecimiento económico, recientemente se ha enfocado hacia los modelos de crecimiento endógeno, en donde la dinámica del crecimiento es independiente de las políticas públicas, y en donde estas, tienen efectos sobre el nivel, pero no sobre el sendero de largo plazo. ${ }^{1}$ Sin embargo, esta no es la forma mas adecuada para entender la dinámica del crecimiento, la racionalización de rendimientos crecientes, constantes o decrecientes de los factores y en especial del capital; lo anterior, nos lleva al cuestionamiento de toda la literatura, sobre la maximización de beneficios y la utilización de factores productivos.

Por otro lado, la estimación de funciones de exportaciones e importaciones conlleva un planteamiento sobre los supuestos del comercio internacional. La visión tradicional, establece que la cantidad de bienes de importación es determinada por las condiciones de demanda doméstica, mientras que la cantidad de bienes de exportación es ocasionada por condiciones de la demanda externa; este enfoque supone la existencia de un país pequeño que actúa como tomador de precios en el mercado internacional.

No obstante, este procedimiento es incorrecto, cuando se consideran los bienes de importación y exportación del mismo país. Tradicionalmente, en el enfoque keynesiano se especifica a la estimación de importaciones, como determinada por los factores domésticos y las exportaciones determinadas por los factores externos de la demanda, ${ }^{2}$ es decir el resto del mundo se vuelve tomador de precios.

Al respecto, cabe señalar que en este caso se esta considerando a las importaciones y exportaciones del mismo país; en este sentido, en cuanto a la demanda de importaciones, el país actúa como tomador de precios, pero en cuanto a las exportaciones sería debatible el supuesto de que el resto del mundo es tomador de precios. En base a lo anterior se planteo la demanda de importaciones suponiendo que la elasticidad de oferta de las importaciones sea muy alta (el supuesto del país pequeño), mientras que la oferta de exportaciones la elasticidad de la demanda del resto del mundo por las exportaciones mexicanas es muy alta, por ello se especificó asumiendo que se trata de una función de producción. ${ }^{3}$

Si el país, es en realidad pequeño, uno podría esperar que éste actuara como un tomador de precios en ambos tipos de bienes importaciones y exportaciones; en donde la demanda de bienes de importación es determinada por las condiciones domésticas de la demanda, en tanto que los bienes de exportación son determinados por las condiciones domésticas de la oferta.

La demanda de bienes comerciables usualmente, se especifica como una función lineal o log lineal, en términos del producto o ingreso real, del precio relativo de los bienes comerciables internacionalmente y el precio doméstico de los bienes substitutos; dependiendo, si los bienes comerciables internacionalmente son considerados como bienes intermedios o como productos finales. Esto es posible, solo si las importaciones y los bienes domésticos pueden ser agregados;

1 R. Barro y J. Sala I. Martín (1995).

2 Para mayor detalle véase E. E. Leamer and R. M. Stern (1970).

3 Una explicación mas extensa se encuentra en J. Tapia y J. Cervantes (1992). 
tales funciones, de demanda de importaciones pueden ser, a su vez derivadas de la función de producción convencional o por la teoría de la utilidad.

El supuesto de agregación de "Hicks", implica que "la función de utilidad es débilmente separable entre las importaciones y los bienes domésticos o que la tecnología es débilmente separable entre importaciones, insumos o factores productivos domésticos y el producto".

La tecnología es representada por una función restringida de beneficios, con el trabajo y el capital siendo fijos en el corto plazo y los precios de las importaciones, exportaciones, bienes de inversión y consumo exógenos. Es decir de la función del producto, uno puede derivar la función de demanda de importaciones y las funciones de oferta, de exportaciones, de bienes de inversión y de consumo, así como la inversa de la demanda de por trabajo y capital. ${ }^{4}$

De esa forma, el sistema completo es derivado y estimado simultáneamente, imponiendo las restricciones apropiadas de simetría y homogeneidad, sobre la producción de los diferentes tipos de bienes y factores productivos; adicionalmente nos proporciona información sobre las posibilidades de sustitución, inherentes en el conjunto o set de tecnología y por algún cambio tecnológíco. Así mismo, se obtienen estimaciones de las funciones de importaciones y exportaciones, sin suponer a priori, supuestos restrictivos.

\section{Antecedentes}

En relación a este tipo de estudios es oportuno señalar que para el caso de México no existen estudios similares, por la escala del modelo planteado; aunque por un lado, existen algunos sobre oferta y demanda de exportaciones dentro de un contexto keynesiano tradicional; ${ }^{5}$ así como algún modelo econométrico trimestral, ${ }^{6}$ en donde se plantean dos ecuaciones del sector externo- una demanda de importaciones y una oferta de exportaciones- una ecuación para la actividad económica y otra para la inflación; señalando, el efecto de la apertura comercial, en las ecuaciones del sector externo y como afectan estas, al equilibrio interno - actividad económica y precios, evidenciando así una doble causalidad.

Por el otro, el de los grandes modelos macroeconómicos de los 80 's para la economía mexicana de corte keynesiano, con periodicidad anual dentro de los que se encuentran los modelos de SEPAFIN (Secretaría de Patrimonio y Fomento Industrial) de A. de Oteyza, el Plan Global de Desarrollo de SPP (Secretaría de Programación y Presupuesto) de R. Montemayor S. y trimestrales como el de J. R. Amieva, Comparación del Poder de Predicción de un modelo

4 Este enfoque tiene alguna remembranza con el tratamiento de D. K. Foley and M. Sidrauski (1970). Portafolio choice, investment, and growth. American Economic Review, 60 , pp. 44-63; en cuanto al tratamiento de la función de producción y la función de producción de los bienes de inversión. La mayor diferencia es la inclusión de las importaciones y exportaciones y el relajamiento del supuesto de la producción no conjunta.

5 Como el de J. Salas y J.J. Sidaoui (1983).

6 Boletín de Economía Internacional, Vol. XIV, Núm. 4, Oct-Dic. 1988. México un Modelo Econométrico del Impacto de la Apertura Comercial en la Balanza Comercial, Actividad Económica y Precios, de J. Tapia M. y Jesús Cervantes. 
Macroeconómico. El Trimestre Económico y el modelo: Desarrollo y Perspectivas de la Economía Mexicana en el Corto Plazo: Un Modelo Econométrico Trimestral, de F. Clavijo. El Trimestre Económico, ambos de gran escala.

En este sentido, la utilidad es la de contar con un modelo mensual sobre el indicador global de la actividad económica a partir de información de indicadores mensuales por el lado de la demanda agregada especificados como funciones de producción; en donde se puedan elaborar políticas de precios y de dotación de factores acordes con una estrategia de crecimiento industrial, con sus consecuentes efectos de política económica, dada la sustituibilidad y complementariedad de los sectores y factores especificados.

Derivado de lo anterior se podría contribuir: primero, al análisis y al establecimiento de proyecciones sectoriales y de uso de factores; segundo, a resaltar la importancia de la estrategia de precios de bienes de consumo, inversión y/o precios relativos (el tipo de cambio real y precios de los bienes comerciables internacionalmente), al cuantificar los efectos que se tendrían a nivel sectorial; tercero, a partir por ejemplo, de una menor demanda externa, el crecimiento se podría impulsar con la producción del mercado interno y viceversa si cae el mercado interno se podría utilizar el sector externo para mantener el crecimiento objetivo de la economía; cuarto, se podría contribuir al diseño de políticas conducentes a fomentar la dotación de factores productivos a diferentes sectores a fin de obtener alguna especialización, tanto de la producción interna como la del sector externo; quinto, una mejor utilización de los factores productivos, a partir de los efectos de sustitución y complementariedad dentro de las funciones de producción, incrementando la eficiencia económica.

El trabajo tiene como objetivo fundamental analizar las posibilidades de sustitución entre las importaciones, exportaciones y la producción de bienes de consumo y de inversión, así como de insumos domésticos y externos, empleando fundamentos básicos de la teoría de la producción; en donde para su implementación empírica se utilizó la herramienta econométrica.

Como está organizado el artículo, este se encuentra formado de la siguiente manera: En la primera parte, se encuentra la introducción en donde se plantean algunas interrogantes de la literatura económica sobre este tema; en la segunda se describe el modelo a utilizar, en términos de la teoría de la producción; en la tercera parte se encuentra la implementación empírica en donde se enuncia la forma funcional, la especificación estocástica y la técnica de estimación, en la cuarta parte se presentan los resultados empíricos con los resultados obtenidos y finalmente en las conclusiones se llevan a cabo comentarios de índole general y sobre aspectos que podrían considerarse en otras investigaciones.

\section{El Modelo}

Se supone que las decisiones de importar y exportar son en base a un proceso de maximización de beneficios por parte de las firmas, las cuales operan bajo un esquema competitivo, en los mercados de factores y de bienes. Las firmas escogen su nivel óptimo de producción y sus requerimientos de importaciones, sujetas al vector de producto y precios de importaciones, así como a la dotación inicial de factores productivos primarios internos. Estos factores internos se asume que tienen movilidad entre las firmas y que sus precios de referencia, son determinados por su producto marginal. 
También se considera que la tecnología es del tipo " $J$ " no negativa para los factores productivos internos - fijos en el corto plazo- y cantidades variables de producto e importaciones " $I$ ". El producto -output- se describe como una variable, positiva en sus cantidades y negativa en importaciones. Se denota al vector de factores productivos o inputs por " $x$ ", a la cantidad variable como " $y$ " $\mathrm{y}$ a los vectores de precios correspondientes, por " $w$ " y " $p$ " respectivamente.

La posibilidad de producción " $T$ " se define como el conjunto de todas las combinaciones posibles de factores productivos y producto "input-output". Se considera que, el conjunto de tecnología agregado satisface el supuesto de rendimientos constantes a escala - $\mathrm{RCE}$-, disposiciones libres, tasas marginales de sustitución y transformación no incrementales, para una dotación de factores fija y el producto u output es variable en cantidades finitas. ${ }^{7}$

Bajo estas condiciones y ya que se supuso una maximización de beneficios, el equilibrio competitivo, puede también caracterizarse en cualquier punto en el tiempo, como la solución al problema de maximización del producto sujeto al conjunto tecnológico, la dotación inicial de recursos domésticos, y a un vector positivo de producto u output y de precios de importación. La tecnología puede entonces, ser representada por una función de beneficio restringida del producto definida como:

$$
\pi(p ; x)=\max _{y}\left\{p^{\prime} y:(x ; y) \in T, p>>0\right\} .
$$

Donde $\pi$ es una función real extendida y esta bien definida para todos los vectores positivos de precios $p$. Bajo el supuesto hecho sobre "T" , la función de beneficios restringida, es linealmente homogénea, monótonamente creciente y cóncava en las cantidades de insumos fijos. También es linealmente homogénea y convexa, en los precios de las cantidades que son variables y monótonamente decreciente o creciente, en estos precios, dependiendo de cuando la cantidad es una importación o un producto u output.

Si la función de beneficio restringida, es diferenciable en $p^{*}$ y $x^{*}$ con respecto de los componentes de $p$, las ecuaciones derivadas de las demandas y las ofertas para las cantidades variables pueden ser obtenidas por diferenciación; un resultado conocido de esto, es el "lema de Hotelling".

$$
y\left(p^{*} ; x^{*}\right)=V_{P} \pi\left(p^{*} ; x^{*}\right),
$$

donde, $V$ es el operador del diferencial del vector, $V_{p} \pi\{p ; x)$ es el vector de las diferencias de primer orden -el gardiente- de $\pi$ con respecto a los componentes de $p$. Similarmente, si la función de beneficio restringido es diferenciable en $p^{*}$ y $x^{*}$ con respecto de los componentes de " $x$ ", entonces el inverso de las funciones de demanda para los factores productivos primarios domésticos, pueden derivarse de la siguiente manera:

$$
w\left(p^{*} ; x^{*}\right)=V_{s}\left(p^{*} ; x^{*}\right),
$$

$7 T$ es un subconjunto de espacio dimensional de $I+J$ y se supone que es no vacío, cerrado, convexo en relación a los componentes de la vecindad de $y$ desde arriba, para un conjunto de $x$ fijas. 
Después de especificar la forma funcional de la función de beneficios restringida, el sistema de ecuaciones de oferta y demanda, puede ser derivado y estimado conjuntamente, para determinar las posibilidades de sustitución implícitas en el set de tecnología.

La tecnología aquí descrita, es de alguna manera similar a la tecnología subyacente en el modelo Heckscher-Ohlin-Samuelson -HOS- el modelo de teoría pura del comercio internacional. En ese modelo, HOS, 2 bienes se producen separadamente, por 2 funciones de producción, utilizando 2 factores productivos, que satisfacen las condiciones de regularidad y dada la dotación inicial de factores domésticos, la cantidad de los 2 bienes producidos es únicamente determinada por los precios relativos del producto.

Mass aún, considerando que la especialización no existe, el vector de precios del producto, también determina los precios de las rentas, por el uso de factores. La mayor diferencia entre el modelo HOS y el aquí propuesto con relación a la tecnología, es que en este último se supone que los bienes son producidos conjuntamente. Entonces, no hay necesidad, para que el número de variables de cantidades sea igual al número de factores productivos, para asegurar que el equilibrio sea determinado cuando no exista la especialización. Una vez que se ha relajado el supuesto de producción separada o no-conjunta, no obstante, los precios de las rentas de los factores, como costo de uso, serán función no solo de los precios de las cantidades variables, sino también de la dotación de factores domésticos.

Ya que, las decisiones de importaciones y exportaciones, se consideran, que son llevadas a cabo por firmas individuales maximizadoras de precios, la balanza comercial será fortuita. El modelo presentado aquí, es de equilibrio parcial y no incluye los mecanismos de ajuste de balanza de pagos. ${ }^{8}$

Escribiendo el Hessiano de la función de beneficios como:

$$
H=\left[\begin{array}{cc}
\pi_{p p} & \pi_{p x} \\
\pi_{x p} & \pi_{x x}
\end{array}\right]=\left[\begin{array}{cc}
V_{p p}^{2} & V_{p x}^{2} \pi \\
V_{x p}^{2} \pi & V_{p p}^{2} \pi
\end{array}\right],
$$

donde $V_{p p}^{2} \pi$ denota la matriz de diferencias de segundo orden de $\pi(p ; x)$ con respecto de los componentes de $p$, la matriz de sustitución $\sum$ puede ser definida como:

$$
\sum=\left[\sigma_{m n}\right]=\left[\begin{array}{ll}
\sum_{p p} & \sum_{p x} \\
\sum_{x p} & \sum_{x x}
\end{array}\right]=\pi\left[\begin{array}{lll}
\pi_{p}^{-1} \pi_{p p} \pi_{p}^{-1} & \pi_{p}^{-1} \pi_{p x} \pi_{x}^{-1} \\
\pi_{x}^{-1} \pi_{x p} \pi_{p}^{-1} & \pi_{x}^{-1} \pi_{x x} \pi_{x}^{-1}
\end{array}\right],
$$

donde $\pi_{p}=\operatorname{diag}\left(V_{p}(p ; x)\right)$ y $\pi_{x}=\operatorname{diag}\left(V_{x} \pi(p ; x)\right)$.

Los elementos de $\sum_{p p}$ son las elasticidades parciales de transformación entre las variables de cantidades, y los elementos de $\sum_{x x}$ son el inverso de las elasticidades parciales de sustitución entre los insumos domésticos; los elementos de

8 Cualquier superávit o déficit de la balanza comercial resultado de una maximización de beneficios en el sector productivo, se supone, que es acomodado por un flujo de capitales, las reservas internacionales o por el mercado de cambios. 
$\sum_{p x}$ y $\sum_{x p}$ son también llamados las elasticidades de intensidad, y la simetría de $\sum$ es implícita directamente, por la simetría del Hessiano. Adicionalmente, la curvatura de las propiedades de la función de beneficios restringida implica, que $\sum_{p p}$ es positiva semi-definida y que $\sum_{x x}$ es negativa semi-definida.

Ahora, podemos definir la matriz de elasticidades-precio de las cantidades variables y las elasticidades de factores o inputs de cantidad domésticos como sigue:

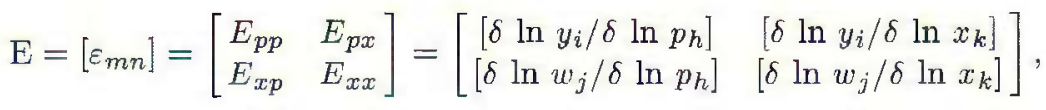

$$
\begin{aligned}
& m, n=1, \cdots, l+J ; \quad i, h=1, \ldots, l ; \quad j, k=l+1, \cdots, l+J .
\end{aligned}
$$

Puede ser demostrado que: ${ }^{9}$

$$
\varepsilon_{m i}=\sigma_{m i}\left(p_{i} y_{i} / \pi\right), \quad m=1, \cdots, l+J, \quad l=1, \cdots, l,
$$

y que

$$
\varepsilon_{m j}=\sigma_{m j}\left(w_{i} x_{j} / \pi\right), \quad m=1, \cdots, l+J, \quad l=1, \cdots, l+J
$$

Los elementos de $E_{P P}$ son las elasticidades parciales de precios del producto ofrecido y de la demanda de importaciones, y los elementos de $E_{x x}$ son el inverso de las elasticidades parciales de precios de la demanda por factores domésticos. ${ }^{10}$

Los componentes de $E_{p x}$ indican, el efecto relativo de un cambio en la dotación inicial de factores domésticos en la demanda y oferta de cantidades variables, y los elementos de $E_{x p}$ indican, el efecto relativo de un cambio en la variable de los precios de cantidades del factor de precios de rentas. Las propiedades de homogeneidad de la función de beneficios restringida, implican que los renglones de $E_{P P}$ y $E_{x x}$ suman cero, mientras que $E_{p x}$ y $E_{x p}$ suman uno. ${ }^{11}$

9 Por definición, E puede escribirse como $\mathrm{E}=\left[\begin{array}{ccc}\pi_{p}^{-1} \pi_{p p}^{-1} & p & \pi_{p}^{-1} \pi_{x x} x \\ \pi_{x}^{-1} \pi_{x p}^{-1} & p & \pi_{x}^{-1} \pi_{x x} x\end{array}\right]$ donde $P=$ $\operatorname{diag}(p)$ y $X=\operatorname{diag}(x)$, se tiene que: $\mathrm{E}=\pi^{-1}\left[\begin{array}{lll}\sum_{p p} \pi_{p} & p & \sum_{p x} \pi_{x} x \\ \sum_{x p} \pi_{p} & p & \sum_{x x} \pi_{x} x\end{array}\right]$. Es conveniente usar este resultado, al computar $\mathrm{E}$, porque $\sum$ puede derivarse, cuando la forma funcional Translog es utilizada.

$10 \sum_{p p}$ siendo positivo, semidefinido, implica que los elementos de su diagonal, $\sigma_{i i}$ es la elasticidad parcial de transformación, de la variable de cantidades, donde todas son no negativas, $\varepsilon_{i i}$ es la elasticidad precio parcial de la variable de cantidades, $i$ es entonces no negativa o no positiva, dependiendo de si la variable de cantidad $i$ es un producto neto o una importación( $y_{i}$ siendo entonces negativa).

11 Cabe señalar, que por medio de la teoría de la dualidad, se podría llevar a cabo una estimación de la función de costos y a través del Lema de Sheppard, obtener la minimización de costos de la demanda de factores. 


\section{Implementación Empírica}

\subsection{Forma Funcional}

Para representar la función del PIB, se requiere una forma funcional lo suficientemente flexible, que no restrinja a priori los signos o la magnitud de las elasticidades de sustitución. La función transcendental (Christensen, Jorgenson y Lau) se ajusta a este proceso y es una serie de expansión de Taylor, logarítmica de segundo orden, para la función restringida de beneficios y se puede escribir así:

$$
\begin{aligned}
& \ln \pi=\alpha_{0}+\sum \alpha_{i} \ln p_{i}+\sum \beta_{j} \ln x_{j}+\frac{1}{2} \sum \sum \gamma_{i h} \ln p_{i} \ln p_{h} \\
& +\sum \sum \delta_{i j} \ln p_{i} \ln x_{j}+\frac{1}{2} \sum \sum \phi_{j k} \ln x_{j} \ln x_{k},
\end{aligned}
$$

donde $i, h=1, \cdots, l ; j, k=l+1, \cdots, l+J$. La simetría del Hessiano de la función de beneficio restringida, implícita en el teorema de Young requiere que:

$$
\gamma_{i h}=\gamma_{h i}, \quad \phi_{j k}=\phi_{k j} .
$$

Adicionalmente, se supone que la función restringida de beneficio, es linealmente homogénea en los precios de las variables de cantidad y en los factores productivos domésticos. Lo anterior requiere que:

$\sum \alpha_{j}=1, \quad \sum \beta_{j}=1, \quad \sum \gamma_{i h}=0, \quad \sum \phi_{j k}=0, \quad \sum_{i} \delta_{i j}=0, \quad \sum_{j} \delta_{j i}=0$.

Diferenciando logaritmicamente la función restringida de beneficios, las ecuaciones de las variables de cantidades de las ofertas y los factores inversos de las ecuaciones de demanda son derivadas, es decir:

$$
\begin{gathered}
V_{i}=\left(y_{i}\right)=\left(\delta \ln p_{i} y_{i}\right)=\alpha_{i}+\sum \gamma_{i h} \ln p_{h}+\sum \delta_{i j} \ln x_{j}, \\
U_{j}=\left(x_{j}\right)=\left(\delta \ln w_{i} x_{j}\right)=\beta_{j}+\sum \delta_{i j} \ln p_{i}+\sum \phi_{j k} \ln x_{k},
\end{gathered}
$$

donde $i, h=1, \cdots, l ; j, k=l+1, \cdots, l+J$. Las $V^{\prime} s$ son entonces el vector con respecto del producto de las variables de cantidades (las importaciones son negativas) y las $U^{\prime} s$ son las utilizaciones de los factores domésticos. La homogeneidad lineal en precios implica que las $V^{\prime} s$ sumadas sean la unidad y la homogeneidad lineal en factores domésticos implica también las $U^{\prime} s$ en conjunto sumen uno.

Una ventaja de la forma funcional de la función Translog ${ }^{12}$ es que la sustitución de la matriz estimada $\sum$ puede ser computada fácilmente, los elementos de $\sum_{p p}$ por ejemplo puede ser calculada como sigue:

$$
\sigma_{i h}=\frac{\pi\left(\delta^{2} \pi / \delta p_{i} \delta p_{h}\right)}{\left(\delta \pi / \delta p_{i}\right)\left(\delta \pi / \delta p_{h}\right)}=\frac{\gamma_{i h}+V_{l} V_{H}}{V_{l} V_{H}}, \quad i, h=1, \cdots, l, \quad i \neq h
$$

12 Es decir que son muy flexibles de tal manera que no se restringe a priori los signos o la magnitud de las elasticidades de sustitución. 


$$
\sigma_{i i}=\frac{\pi\left(\delta^{2} \pi / \delta p_{i}^{2}\right)}{\left(\delta \pi / \delta p_{i}\right)^{2}}=\frac{\gamma_{i i}+V_{l}^{2}-V_{i}}{V_{l}^{2}}, \quad i=1, \cdots, l .
$$

Esto es aplicable para otros elementos de $\sum$.

Hasta ahora no se ha permitido cambio tecnológico, sin embargo es claro que las ecuaciones de demanda y oferta son consistentes con el cambio tecnológico a la "Hicks", neutral o de cambio cualitativo, tanto en factores, como en productos. ${ }^{13}$ Se puede demostrar entonces que, si se desea permitir cambio tecnológico en factores o en productos, se debe incluir una variable de tendencia, para mayor detalle véase (Kohli, 1975). Por lo que, se puede probar la significancia estadística de esta última variable de tendencia en la determinación anterior, para probar la hipótesis de cambio tecnológico neutral a la Hicks, la cual se puede incluir como un caso especial.

\subsection{Especificación estocástica y técnicas de estimación}

Si suponemos que la función Translog ${ }^{14}$ del producto es una representación exacta de la tecnología y que cualquier desviación de los factores y el producto de la maximización de beneficios son debidas a los errores en la optimización y son aleatorios; especificamos entonces un vector de errores aleatorio.

$$
\left(v_{i}^{\prime}, u_{i}^{\prime}\right)^{\prime}=\left(v_{1 i}, \cdots, v_{l i}, u_{l+1}, \cdots, u_{l+J}\right)^{\prime} ; \text { tal que } \sum_{i}^{l} v_{i}=0, \sum_{i+1}^{l+J} u_{j}=0
$$

Las $V^{\prime} s$ y las $U^{\prime} s$ son vectores que se suponen idénticamente distribuidos- normales y aleatorios- con media cero y con matriz de covarianza $\Omega$. Ya que la variable de cantidades, así como la de factores domésticos suman uno, la matriz de covarianza es singular y por lo tanto 2 ecuaciones una de cada lado deben de ser eliminadas para propósitos de estimación. Sin embargo, la estimación no depende de cual de las 2 ecuaciones son eliminadas (Barten, 1969). Vale la pena considerar la posibilidad de autocorrelación de primer orden; los elementos de la matriz de coeficientes de autocorrelación de primer orden no pueden ser identificados en el caso general, porque la matriz de covarianza es singular (Berndt and Servin, 1975).

Por simplicidad, solo se consideró, el caso en donde los coeficientes de la matriz de autocorrelación de primer orden es diagonal, esto implica que todos los coeficientes de autocorrelación de las variables de cantidad por un lado y los de los factores domésticos por el otro deben de ser idénticos (Berndet and Servin, 1975). Los coeficientes de autocorrelación de las ecuaciones de ofertas de cantidades variables y las correspondientes a las ecuaciones de los inversos de las demandas de insumos fijos se denotan por $\rho_{v} \mathrm{y} \rho_{u}$ respectivamente.

13 En cuanto a la posibilidad de un cambio tecnológico sesgado, se incluyó el cambio tecnológico neutral por simplicidad del análisis, aunque desde luego que podría intentarse el cambio tecnológico sesgado, aunque el planteamiento general tendría que ser modificado en términos de utilización de factores y en la función de producción sectorial.

14 En este sentido la forma funcional seleccionada proporciona una velocidad de ajuste hacia los valores de equilibrio de largo plazo muy alta. 
Cada ecuación puede ser escrita como una función de las variables exógenas del período corriente y con algún rezago, así como de la utilización de insumos o productos, pero el sistema se convierte en uno no-lineal en los parámetros. ${ }^{15}$ Desde luego que el sistema es identificado de acuerdo a las condiciones de orden y de rango y se presentan tan solo las formas reducidas.

A fin de corroborar la hipótesis del crecimiento endógeno, en donde la dinámica del crecimiento es independiente de las políticas públicas, se llevó a cabo un análisis de estas series, con el objeto de ver si son estacionarias, por lo que se hicieron pruebas de Dickey-Fuller, resultando positivas de orden uno. Desde luego que, dado el conjunto de información se ajustaron las series y adicionalmente se consideraron diferencias de orden 12 para hacerlas estacionarias.

Así mismo, se eliminó de la relación de comportamiento del producto, el gasto gubernamental y en cuanto a los insumos o factores productivos, solo se tomó en cuenta el trabajo y el capital nacional e importado. ${ }^{16}$

Dado que el Método Generalizado de Momentos, es un estimador robusto que no requiere información exacta de la distribución de los errores, se llevó a cabo esta estimación simultánea cuyos resultados se presentan posteriormente. Así mismo, se emplearon, estimaciones simultaneas del sistema por medio de Mínimos Cuadrados en Tres Etapas, que permite al sistema ser no-lineal en los parámetros; aunque si el sistema es lineal, el algoritmo es similar al método de Zellner (1962) Seemingly Unrelated Regression Equations, por lo que también se estimó el sistema con este método, para examinar la validez empírica de la hipótesis alternativa, en cuanto a que los resultados del modelo general pudieran obtenerse de un mejor estimador como los anteriormente mencionados, a través de una prueba Non-nested Test.

\subsection{Descripción de los datos}

El modelo descrito anteriormente es usado para estimar la estructura de la tecnología mexicana en logaritmos con el operador $(L)$, en un período mensual de enero 1993 a junio de 2003, con relación a los datos que se utilizaron, como aproximación del producto o PIB mensual, el IGAE (Indicador Global de Actividad Económica) ( $\left.L_{y}\right)$ del INEGI ${ }^{17}$ y se propuso la descomposición de éste, a partir de los componentes de la demanda agregada aproximados por: 1) Los bienes de Consumo, por el índice de la producción de Bienes de Consumo $\left(L B^{C}\right)$, los cuales incluyen los bienes de Consumo Duradero y No-Duradero del INEGI, 2) Los bienes de Inversión, por el Indicador Mensual de la Formación Bruta de Capital Fijo $\left(L B^{I}\right)$ del Banco de México, desagregado en sus componentes de Maquinaria y Equipo y Construcción- descontadas las Importaciones

\footnotetext{
15 Para mayor detalle vease la restricción de Wald en la demanda de factores capital interno y externo del apendice $C$.

16 Se pueden incorporar desde luego otros factores como electricidad, para mayor detalle véase R. Pindyck and J. Rotemberg (1983). Dynamic Factor Demands and the effects of Energy Price Shocks. American Economic Review, pp. 1066-1079.

17 Como aproximación del PIB mensual, se utilizó el IGAE, por lo que esta se refiere tanto a la producción de manufacturas, como la de los sectores agropecuario, comercio y servicios; en las manufacturas se incluyen las industrias de transformación, extractivas, construcción, electricidad, gas y agua.
} 
de Maquinaria y Equipo importado-, 3) Las Exportaciones $\left(L B^{X}\right)$ e Importaciones totales $\left(L B^{M}\right)$ en pesos reales con los indicadores de Comercio Exterior de Banco de México. Los insumos primarios domésticos son trabajo medido como las horas hombre trabajadas en el sector manufacturero $\left(L H^{H T}\right)$ del INEGI y capital interno $\left(L K^{I}\right)$ y externo $\left(L K^{E}\right)$, a través de la formación bruta de capital fijo $K$ (producción de maquinaria y equipo nacional e importado) del Banco de México. Los precios de las los bienes comerciables internacionalmente $\left(L P^{B C}\right.$ ), (aunque en algunos casos se utilizó el tipo de cambio real $\left(L T C^{R}\right)$, de los bienes de consumo privado $\left(L P^{C}\right)$ y de capital $\left(L P^{I}\right)$, (formación bruta de capital fijo), a través de sus índices de precios respectivos publicados por el Banco de México. ${ }^{18}$

La función de producción restringida, puede ser calculada por :

$$
\Pi=p_{c} y_{c}+p_{i} y_{i}+p_{x} y_{x}+p_{m} y_{m}
$$

Respecto de la ecuación de la aproximación del PIB mensual -IGAE- se decidió incluir solo estas variables, ya que de otra forma, se tendría el problema de correr una identidad, y desde luego el problema de correlación serial entre las ecuaciones, puesto que los errores de unas ecuaciones se compensarían con otros.

Adicionalmente, es claro, que México produce muchos productos terminados, los cuales podrían ser igualmente exportados o dirigidos al mercado interno; esto parece ser difícil de justificar, desde el punto de vista, de que las exportaciones, así como los bienes de producción para consumo o inversión suelen ser agregados; uno puede argüir sin embargo, en el contexto del presente modelo, que al menos en el sentido de que los bienes de consumo y los que se exportan son conceptualmente diferentes ya que usan diferentes canales de distribución. Esta dificultad no se presenta con los bienes intermedios, ya que estos al salir de la producción interna, son exportados.

Por otro lado, las ecuaciones de oferta sectorial son derivadas de la ecuación (7), representada por la forma funcional de acuerdo a la función Transcendental de Christiansen, Jorgenson y Lau ajustada por medio de una expansión de Taylor, que diferenciándola obtenemos las ecuaciones (8) y (9) que serían las formas reducidas a estimar del resto del sistema

\section{Resultados empíricos}

Después de eliminar las ecuaciones del consumo de gobierno con el propósito de estimación e imponiendo las restricciones de simetría y homogeneidad, el sistema a estimar es: ${ }^{19}$

18 Intentos para desagregar las importaciones y exportaciones o la inclusión de variables adicionales como tasas impositivas pueden llevarse a cabo, aunque si el número de demandas derivadas y ecuaciones de oferta se incrementan, la estimación simultanea se dificulta; dadas las condiciones de curvatura que se deben de imponer como puntos de expansión.

19 Se aclara que no se ponderaron las variables independientes con relación al valor del producto total ya que provienen de diferentes indicadores y el propósito es ver si esos son estimadores correctos para su agregación al menos en su composición y si estos a su vez se pueden desagregar. 


$$
\begin{aligned}
& L_{y}=\alpha_{1}+\alpha_{2} L B^{c}+\alpha_{3} L B^{l}+\alpha_{4} L B^{X}+\alpha_{5} L B^{M}+\alpha_{6} t+\alpha_{7} L y_{t-1}+V_{1 t}, \\
& L B^{C}=\alpha_{8} L H^{H T}+\alpha_{9} L K^{E}+\alpha_{10} L P^{C}+\alpha_{11} L P^{l}+\alpha_{12} L T C^{R}+\alpha_{13} L B_{t-1}^{C}+U_{1 t} \\
& L B^{l}=\alpha_{14}+\alpha_{15} L H^{H T}+\alpha_{16} L K^{E}+\alpha_{17} L K^{l}+\alpha_{18} L P^{C}+\alpha_{19} L P^{l} \\
& \quad+\alpha_{20} L P^{B C}+\alpha_{21} L B_{t-1}^{l}+U_{2 t}, \\
& L B^{X}=\alpha_{22} L H^{H T}+\alpha_{23} L K^{E}+\alpha_{24} L P^{C}+\alpha_{25} L P^{l}+\alpha_{26} L T C^{R}+\alpha_{27} t \\
& \quad+\alpha_{28} L B_{t-1}^{X}+U_{3 t}, \\
& L B^{M}=\alpha_{29} L H^{H T}+\alpha_{30} L K^{l}+\alpha_{31} L P^{C}+\alpha_{32} L P^{l}+\alpha_{33} L T C_{t-1}^{R} \\
& \quad+\alpha_{34} L B_{t-1}^{M}+U_{4 t} .
\end{aligned}
$$

Además, se agregan las siguientes ecuaciones de insumos o factores productivos:

$$
\begin{aligned}
L H^{H T} & =\alpha_{35}+\alpha_{36} L K+\alpha_{37} L K^{l}+\alpha_{38} L P^{C}+\alpha_{39} L P^{l}+\alpha_{40} L T C^{R}+U_{5 t}, \\
L K^{l} & =\alpha_{41}+\alpha_{42} L K^{E}+\alpha_{43} L H^{H T}+\alpha_{44} L P^{C}+\alpha_{45} L P^{l}+\alpha_{46} L T C^{R} \\
& +\alpha_{47} L K_{t-1}^{l}+U_{6 t}, \\
L K^{E} & =\alpha_{48}+\alpha_{49} L K^{l}+\alpha_{50} L H^{H T}+\alpha_{51} L P^{C}+\alpha_{52} L P^{l}+\alpha_{53} L T C^{R} \\
& +\alpha_{54} L K_{t-1}^{E}+U_{7 t} .
\end{aligned}
$$

Y los tẻrminos estocásticos tendrían las siguientes características:

$$
\begin{gathered}
V_{1 t}=\varepsilon_{i}+\rho_{v} V_{i t-1}, \quad \text { con } i=Y \quad \text { y } U_{j t}=\varepsilon_{j}+\rho_{u} U_{j t-1} \\
\text { y } \operatorname{con} j=B^{C}, B^{I}, B^{X}, B^{M}, L H^{H T}, L K^{I}, L K^{E}
\end{gathered}
$$

A continuación se aplicó un operador de diferencia doble logarítmico (DD) antes mencionado, por lo cual los estimadores representan elasticidades y se presentan los resultados obtenidos por el Método Generalizado de Momentos, y en los apéndices A y B, los correspondientes a los métodos de Tres Etapas y Seemly Unrelated Ecuations. ${ }^{20}$

20 Dado que estimadores como Mínimos Cuadrados Ordinarios, Mínimos Cuadrados en Dos Etapas, Variables Instrumentales, Ecuaciones Simultaneas No-Lineales y Modelos de Expectativas Racionales Dinámicas, pueden considerarse casos especiales de el Método Generalizado de Momentos y más aún dentro de este tipo de casos también se puede incluir la estimación de Máxima Verosimilitud, por lo que se optó por este tipo de estimación. En el caso de Estimadores de Tres Etapas este se utilizó por ser considerado asintóticamente eficiente, y finalmente el método de Seemly Unrelated Equations, en donde el supuesto de errores no correlacionados, se prueba como hipótesis alternativa. 


$$
\begin{aligned}
& D D L_{y}=0.004+0.317^{*} D D L B^{C}+0.135^{*} D D L B^{l}+0.037^{*} D D L B^{X} \\
& \begin{array}{llll}
(1.27) & (9.95)^{*} & (9.20)^{*} & (4.93)^{*}
\end{array} \\
& \begin{array}{ccc}
-0.036^{*} D D L B^{M} & +0.00003^{*} T R E N D & +0.265^{*} D D L y_{(-1)} \\
(-3.24)^{*} & (1.09) & (9.21)^{*}
\end{array}
\end{aligned}
$$

R-squared

Adjusted R-squared

S.E. of regression

Durbin-Watson stat
0.946745

0.943702

0.010664

1.779921
Mean dependent var

0.026996

S.D. dependent var

Sum squared resid

0.044945

0.011941

$$
\begin{array}{cccc}
D D L B^{C} & =0.279^{*} D D L H^{H T} & +0.091^{*} D D L K^{E} & +0.182^{*} D D L P^{C} \\
(7.79)^{*} & (6.35)^{*} & (2.02)^{*} \\
& & & \\
-0.084^{*} D D L P^{l} & -0.020^{*} D D L T C^{R} & +0.159^{*} D D L B_{(-1)}^{C} \\
(-0.88) & (-1.00) & (3.47)^{*}
\end{array}
$$

$\begin{array}{llll}\text { R-squared } & 0.705803 & \text { Mean dependent var } & 0.030114 \\ \text { Adjusted R-squared } & 0.691794 & \text { S.D. dependent var } & 0.046331 \\ \text { S.E. of regression } & 0.025721 & \text { Sum squared resid } & 0.069465 \\ \text { Durbin-Watson stat } & \mathbf{1 . 5 0 1 4 3 1} & & \end{array}$

$$
\begin{gathered}
D D L B^{l}=0.034+0.421^{*} D D L H^{H T} \\
\begin{array}{ccc}
(11.04)^{*} & (9.55)^{*} & 0.291^{*} D D L K^{E} \\
& (17.39)^{*} \\
+0.297^{*} D D L K^{l} & -0.360^{*} D D L P^{C} & +0.348^{*} D D L P^{l}- \\
(23.11)^{*} & (-3.17)^{*} & (2.51)^{*} \\
-0.126^{*} D D L P^{B C} & -0.124^{*} D D L B_{(-1)}^{l} & \\
(-4.27)^{*} & (-6.72)^{*}
\end{array}
\end{gathered}
$$

$\begin{array}{llll}\text { R-squared } & 0.988455 & \text { Mean dependent var } & 0.025024 \\ \text { Adjusted R-squared } & 0.987557 & \text { S.D. dependent var } & 0.171658 \\ \text { S.E. of regression } & 0.019148 & \text { Sum squared resid } & 0.032998 \\ \text { Durbin-Watson stat } & \mathbf{1 . 4 2 4 2 4 4} & & \end{array}$




$$
\begin{aligned}
& D D L B^{X}=0.413^{*} D D L H^{H T}+0.101^{*} D D L K^{E}-0.560^{*} D D L P^{C} \\
& (3.70)^{*} \quad(3.47)^{*} \quad(-2.56)^{*} \\
& -1.013^{*} D D L P^{l}+0.764^{*} D D L T C^{R}+0.0000001^{*} T R E N D \\
& (-3.98)^{*} \quad(14.62)^{*} \quad(1.46)^{*} \\
& +0.428^{*} D D L B_{(-1)}^{X} \\
& (11.21)^{*}
\end{aligned}
$$

R-squared

Adjusted R-squared

S.E. of regression

Durbin-Watson stat
0.870941

0.863566

0.078031

1.747665
Mean dependent var

0.101830

S.D. dependent var

0.211255

Sum squared resid

0.639331

$$
\begin{aligned}
& D D L B^{M}=1.244^{*} D D L H^{H T}-0.499^{*} D D L K^{l}+0.098^{*} D D L P^{C} \\
& (10.36)^{*} \quad(-7.36)^{*} \quad(0.47)
\end{aligned}
$$

$$
\begin{array}{lcc}
+0.218^{*} D D L P^{l} & -0.251^{*} D D L T C^{R} & +0.596^{*} D D L B_{(-1)}^{M} \\
(1.02) & (-2.93)^{*} & (16.00)^{*}
\end{array}
$$

$\begin{array}{llll}\text { R-squared } & 0.564456 & \text { Mean dependent var } & 0.087602 \\ \text { Adjusted R-squared } & 0.543313 & \text { S.D. dependent var } & 0.149152 \\ \text { S.E. of regression } & 0.100795 & \text { Sum squared resid } & 1.046443 \\ \text { Durbin-Watson stat } & 2.038887 & & \end{array}$

$$
\begin{gathered}
D D L H^{H T}=-0.041+0.139^{*} D D L K^{E} \\
\begin{array}{c}
(-19.15)^{*} \\
(10.64)^{*}
\end{array} \\
-0.243^{*} D D L P^{C}+0.384^{*} D D L 9^{*} D D L P^{l} \\
(-4.52)^{*} \quad(6.49)^{*} \\
+0.044^{*} D D L T C^{R} \\
(3.29)^{*}
\end{gathered}
$$


R-squared

Adjusted R-squared

S.E. of regression

Durbin-Watson stat
0.840463

0.833008

0.023748

2.005931
Mean dependent var

S.D. dependent var

Sum squared resid

$-0.012096$

0.058115

0.060346

$$
\begin{aligned}
& D D L K^{l}=0.018+0.088^{*} D D L K^{E}+0.939^{*} D D L H^{H T} \\
& (3.04)^{*} \quad(3.41)^{*} \quad(10.11)^{*} \\
& +0.758^{*} D D L P^{C} \quad-0.852^{*} D D L P^{l} \\
& (5.42)^{*} \\
& (-5.12)^{*} \\
& -0.170^{*} D D L T C^{R}+\quad 0.525^{*} D D L K_{(-1)}^{l} \\
& (-4.76)^{*} \\
& (20.12)^{*}
\end{aligned}
$$

R-squared

Adjusted R-squared

S.E. of regression

Durbin-Watson stat
0.923080

0.918726

0.061019

2.126668
Mean dependent var $\quad 0.015390$

S.D. dependent var $\quad 0.214037$

Sum squared resid $\quad 0.394670$

$$
\begin{array}{cccc}
D D L K^{E} & =0.014 & +0.280^{*} D D L K^{l} & +2.427^{*} D D L H^{H T} \\
& (5.71)^{*} & (3.79)^{*} & (10.65)^{*} \\
-1.777^{*} D D L P^{C} & +1.709^{*} D D L P^{l} \\
(-3.81)^{*} & (3.52)^{*} \\
-0.379^{*} D D L T C^{R} & -0.240^{*} D D L K_{(-1)}^{E} \\
(-7.27)^{*} & (3.81)^{*}
\end{array}
$$

$\begin{array}{llll}\text { R-squared } & 0.903068 & \text { Mean dependent var } & 0.049870 \\ \text { Adjusted R-squared } & 0.896946 & \text { S.D. dependent var } & 0.250589 \\ \text { S.E. of regression } & 0.080444 & \text { Sum squared resid } & 0.614772 \\ \text { Durbin-Watson stat } & 1.281444 & & \end{array}$

La prueba "t" de Student aparece entre paréntesis. * Prueba a un extremo. Estadísticamente significativa al 5\%.

Cabe señalar que:

\begin{tabular}{|l|l|}
\hline Determinant residual covariance & $2.16 \mathrm{E}-28$ \\
J-statistics & 0.720730 \\
\hline
\end{tabular}


Estos resultados provienen de los denominados Non-nested Test, en los cuales se contrasta los resultados del modelo en general en el sentido de que pudiera haber otro tipo de estimación, como los que se llevaron a cabo en Three Stages Least Squares y Seemingly Unrelated Regression Equations y se demuestra que la bondad del ajuste obtenido por el Método Genaralizado de Momentos (GMM) es satisfactorio.

\section{Resultados generales del sistema}

Las estimaciones de la función translog de la aproximación del $P I B$ a través del $I G A E$, bajo la hipótesis mantenida de un cambio tecnológico neutral a la Hicks, muestran los signos esperados, aunque la significancia estadística del cambio tecnológico es muy baja. Los reportes convencionales de los estadísticos $R^{2}$ y $D W$, son calculados de los residuales de las ocho ecuaciones y su valor informativo es significativo. Las funciones estimadas satisfacen todas las condiciones de regularidad sobre el rango completo de observaciones de precios y cantidades. Todas las cinco primeras funciones son monotónicamente crecientes en todos sus argumentos, excepto el precio de las importaciones, cóncavas en los inputs primarios domésticos y convexos en las variables de precios de cantidades.

Los diversos resultados de las pruebas "t" estadísticas, reportados al 95\% de nivel de confianza, son satisfactorias; no obstante, la hipótesis de cambio tecnológico a la Hicks en las ecuaciones del producto y las exportaciones es rechazada, por la reducida significancia estadística. ${ }^{21}$ Similarmente, la hipótesis de autocorrelación es rechazada independientemente, de la existencia o no del cambio tecnológico. Los resultados de estas pruebas indican, que en las ocho formulaciones, el modelo de GMM es el que mejor representa al sistema, a partir de la covarianza residual del determinante.

Aquí se presentan las gráficas de los residuales de cada ecuación del sistema, como se puede apreciar los patrones son diferentes en su distribución, amplitud y oscilaciones. ${ }^{22}$

21 Cabe señalar que, en las ecuaciones tanto del $P I B$ como de las exportaciones no fueron significativos, probablemente porque la economía y las exportaciones ya se habían ajustado al modelo de crecimiento con mayor apertura, acentuado desde 1985 con la sustitución de permisos por aranceles y la disminución de las tasas arancelarias, y acelerado desde la firma del TTLC Triple Tratado de Libre Comercio en 1993, aunque la entrada en vigor fuese en 1994.

22 Dado el período de estimación, la firma del TLC se llevó a cabo en 1993, que fue el año de inicio del trabajo, para llevar a cabo pruebas de cambio estructural, se tendría que supone, que este se hubiera llevado a cabo con la firma de otros tratados, en donde se supusiera algún cambio significativo en estructura económica o como resultado del ajuste del vector de precios relativos en 1995, pero los residuales se ajustan muy bien durante el período de estudio; mas aún se llevaron a cabo pruebas del CUSUM (Cumulative Sum of Recursive Residual) con el objeto de buscar alguna inestabilidad de parámetros, si es que la suma acumulada excediera los límite de críticos de confianza al $5 \%$ y cabe señalar que a lo largo del período de estimación, no se presentaron casos extremos. 
Figura 1. DDLY RESIDUALS.

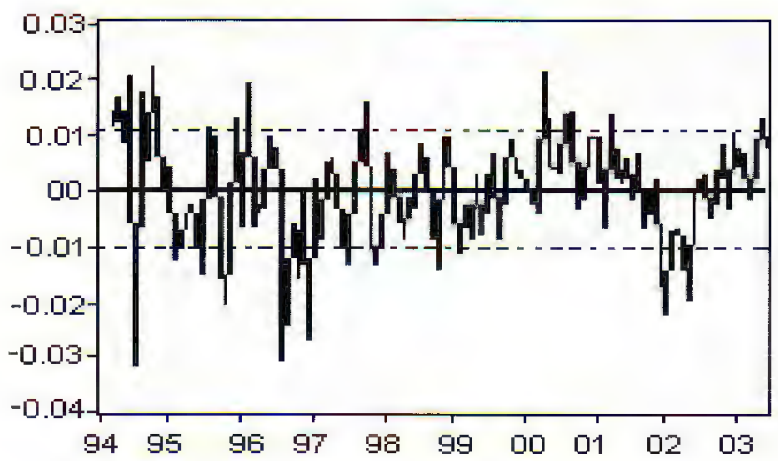

Figura 2. DDLC RESIDUALS.

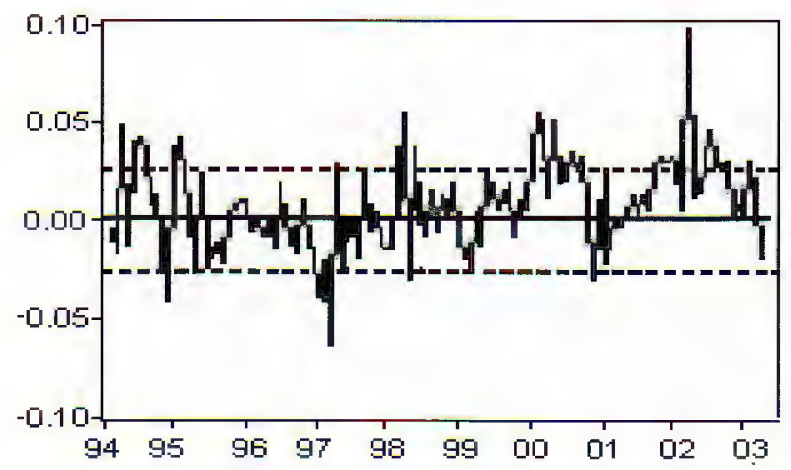

Figura 3. DDLI RESIDUALS.

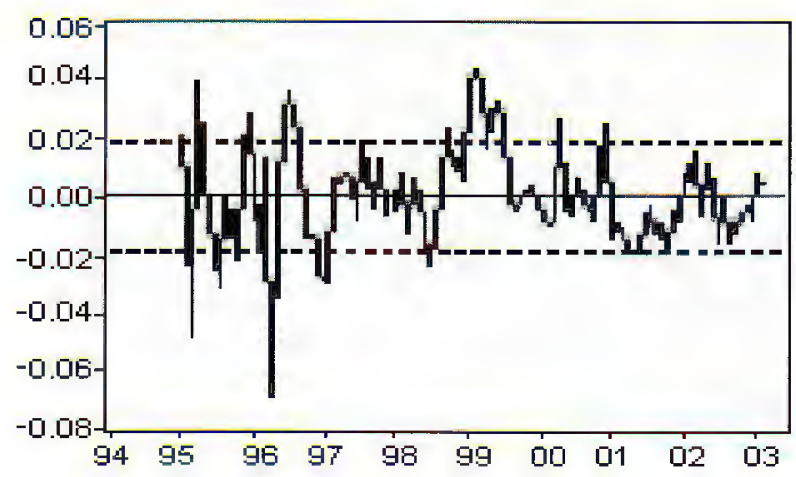


Figura 4. DDLXPR RESIDUALS.

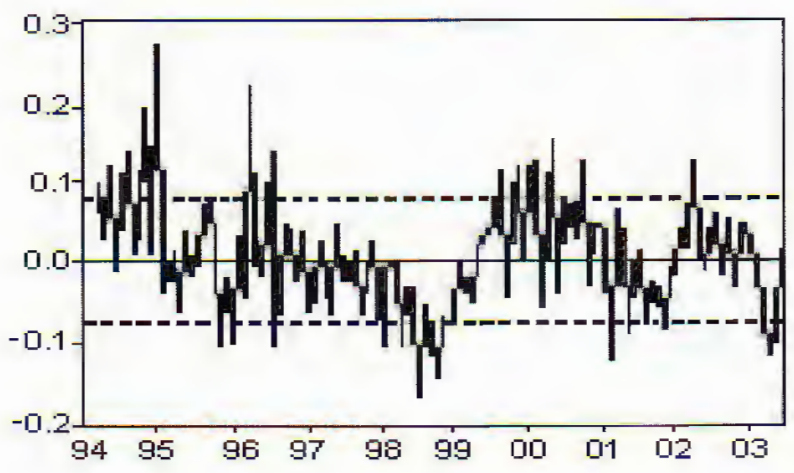

Figura 5. DDLMPR RESIDUALS.

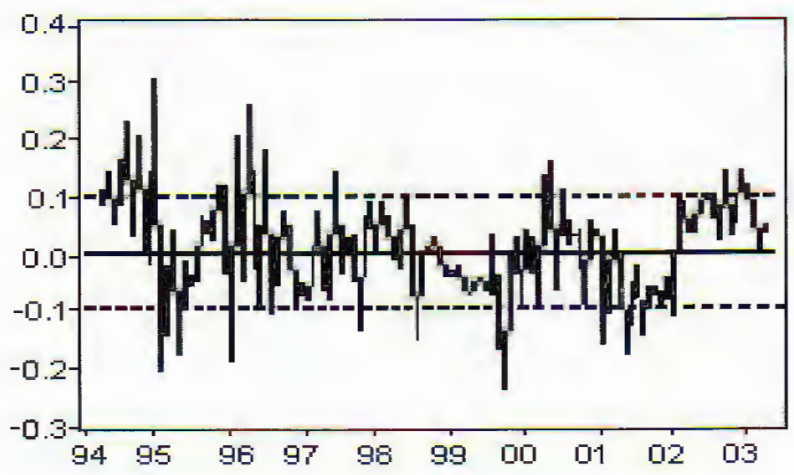

Figura 6. DDLL RESIDUALS.

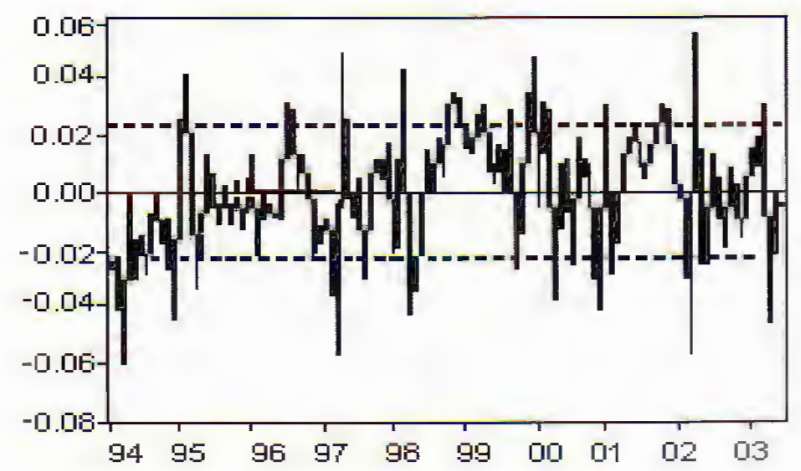


Figura 7. DDLK RESIDUALS.

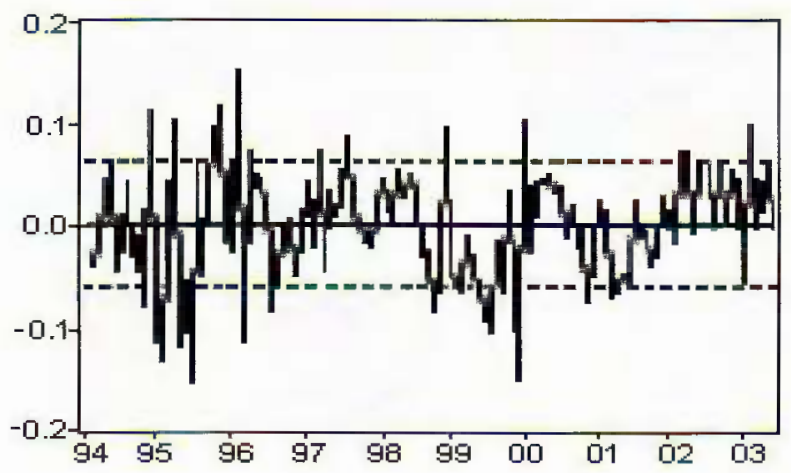

Figura 8. DDLKE RESIDUALS.

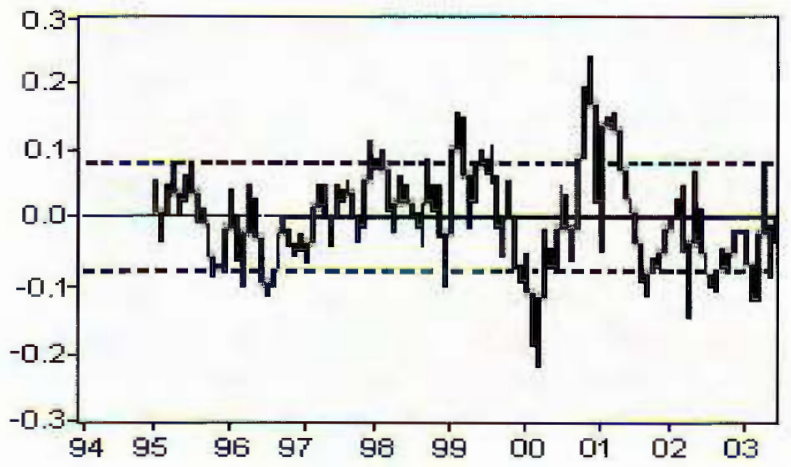

Por otro lado a fin de complementar lo anterior se procedió a calcular la matriz de correlación serial de los errores la cual se presenta a continuación.

Matriz de correlación residual del sistema.

\begin{tabular}{lllllllll}
\hline & DDLY & "LC & "LT & "LXPR & "LMPR & "LL & "LK & "LKE \\
DDLY & 1 & & & & & & & \\
"LC & -0.006 & 1 & & & & & & \\
"LI & -0.009 & -0.10 & 1 & & & & & \\
"LXPR & 0.1463 & 0.266 & -0.12 & 1 & & & & \\
"LMPR & 0.2160 & 0.182 & -0.07 & 0.619 & 1 & & & \\
"LL & -0.111 & 0.471 & -0.00 & -0.09 & --0.26 & 1 & & \\
"LK & 0.1377 & 0.014 & -0.32 & 0.034 & 0.347 & -0.35 & 1 \\
"LKE & -0.010 & -0.40 & 0.177 & 0.025 & 0.035 & -0.33 & -0.20 & 1 \\
\hline
\end{tabular}


Los resultados muestran la bondad del ajuste, ya que esta matriz es simétrica y evidencia que el grado de asociación de los residuales del sistema, es muy aceptable, tal vez con la excepción de la asociación entre los residuales de las exportaciones e importaciones.

\section{Resultados específicos del sistema}

Las matrices de sustitución estimadas no son reportadas aquí, ya que las derivadas parciales de precios y elasticidades de los insumos, reportados los resultados empíricos proveen esencialmente la misma información acerca de las posibilidades de sustitución inherentes a la tecnología mexicana.

En este sentido vale la pena resaltar, que los signos de los componentes de la estimación del producto o PIB por el lado de los usos, son los correctos y significativos al $95 \%$ de confianza; es claro que para ser una estimación mensual guarda una relación estrecha con los componentes de la estructura del mismo, ya que el mas importante es el consumo, seguido de la inversión, las exportaciones y las importaciones del $32 \%, 14 \%, 3.7 \%$ y $3.6 \%$ respectivamente, como se observa en los resultados empíricos; así mismo, la variable de tendencia para explicar el cambio tecnológico neutral a la Hicks no resulto significativo estadísticamente, aunque mostró el signo esperado.

Por otro lado, la producción de bienes de consumo, se incrementa con la mayor utilización de factores productivos resalta la importancia de la estructura del factor trabajo con casi 0.30 y en tanto que la maquinaria y equipo importado representó alrededor del 0.10, es importante señalar que una parte significativa de la producción de los bienes de consumo, son productos que reflejan un uso intensivo de mano de obra y que dada la especificación empleada los rendimientos distan de ser al menos constantes; en cuanto a los precios de los bienes de consumo, la elasticidad estimada indica que al aumentar estos en un $1 \%$, se incrementa su producción en $0.18 \%$, en tanto que los precios de los bienes de inversión y los referentes a los bienes comerciables internacionalmente, medidos por el tipo de cambio real, evidencian la sustitución entre estos tipos de bienes del 0.08 y $0.02 \%$ respectivamente.

En cuanto a la producción de los bienes de inversión, al emplear mas factores productivos mano de obra, maquinaria y equipo interno y externo, mayor es su producción, resalta que a partir de la especificación propuesta los rendimientos se pudieran considerar constantes a escala, ${ }^{23}$ ya que estos suman alrededor de la unidad, y se aprecia que la producción de este tipo de bienes es mas intensiva en el uso del maquinaria y equipo, tanto interno como externo; al incrementar los precios de estos tipos de bienes en un 1\%, los precios de los bienes de inversión, inciden de manera directa en su producción al incrementarse $0.35 \%$, mientras que la de los bienes de consumo y de los bienes comerciables internacionalmente muestran algún grado de sustitución de 0.36 y $0.13 \%$ respectivamente.

En relación a las exportaciones, estas se incrementan, al aumentar los factores productivos y el tipo de cambio real, resalta el uso intensivo de la mano de obra en concordancia con el teorema de Heckscher-Olin en el que se postula que los países exportarán aquellos bienes en los cuales tienen ventajas comparativas

23 Para mayor detalle véase, el apéndice $\mathrm{C}$. 
(un uso intensivo en el factor abundante), dadas la estructuras obtenidas de $0.43 \%$ y $0.10 \%$ para el trabajo y el capital externo respectivamente; mientras que estas se verán reducidas al incrementarse los precios de los bienes de consumo y de inversión en un $1 \%$, en $0.56 \%$ y $1.0 \%$, respectivamente, señalando la sustitución inherente entre estos tipos de bienes. En cuanto a la elasticidad precio estas aumentarán $0.76 \%$, siendo muy sensibles al efecto cambiario.

Mientras que las importaciones dada la estructura productiva planteada, aumentan más que proporcionalmente al incrementarse por el factor trabajo en 1.2 veces y disminuyen al utilizar mas capital en la función de producción en alrededor de 0.50 ; con respecto a los precios al aumentar el tipo de cambio en un $1 \%$, se reducen las importaciones de acuerdo al enfoque de elasticidades en $0.25 \%$, mostrando una elasticidad precio relativamente baja, en tanto que al aumentar los precios de los bienes de consumo y de inversión en 1\%, esto se verá reflejado en 0.10 y $0.20 \%$ respectivamente de incremento, por su menor costo relativo.

Hay que recordar que los precios de los bienes comerciables internacionalmente, bienes de inversión y de consumo, así como las cantidades de trabajo y capital son exógenos, en tanto que sus variables conjugadas estas son endógenas.

Se encontró que la elasticidad parcial del precio de la demanda de importaciones en el largo plazo fluctúa entre -0.6 y -0.8 durante el período muestral y la elasticidad parcial del precio de la oferta de exportaciones en el largo plazo varía entre 1.3 y 1.5. La elasticidad cruzada de precios parcial, muestra que las exportaciones, los bienes de inversión y los bienes de consumo son substitutos entre ellos en la producción, lo que significa que un incremento en el precio del producto de cualquiera de ellos, conduce a una reducción en la oferta de los otros dos; considerando que los precios de los otros bienes e insumos domésticos se mantienen constantes.

Dados los resultados obtenidos, a través de las elasticidades precio de las exportaciones e importaciones un cambio porcentual del tipo de cambio real impulsará mas las exportaciones de lo se reducirán las importaciones.

Asimismo, tanto las exportaciones como los bienes de inversión son dependientes de la maquinaria y equipo importado, en el sentido de que un incremento en el precio de los bienes comerciables internacionalmente y/o el tipo de cambio real estaría acompañado por una caída en la producción de ambos tipos de bienes, o alternativamente que un aumento en el precio de cualquiera de estos 2 tipos de bienes, resultaría en una mayor oferta que se acompañaría de una mayor demanda por importaciones.

Por otro lado, un incremento de en el precio de los bienes de consumo, conduciría a una mayor producción de bienes de consumo pero también a una menor oferta de bienes de inversión y de exportaciones, lo que a su vez induciría a una menor demanda de importaciones.

A fin de estimar los efectos de un cambio en el precio de una variable de cantidad sobre los precios de los factores-rentals-o el efecto de un cambio en la dotación de factores sobre la oferta y demanda de importaciones y de la producción de los diferentes tipos de bienes. Podemos ver entonces que un aumento en los factores productivos como trabajo-horas hombre- o capital incrementarán la producción de bienes de consumo e inversión. Es interesante 
apreciar que un incremento en el flujo de bienes de inversión como maquinaria y equipo podría crear una reducción tanto en las importaciones y un aumento en las exportaciones, con un efecto favorable en la balanza comercial en una condición cercana al equilibrio, ${ }^{24}$ fundamentalmente por la reducción de las importaciones.

Respecto de la demanda de factores, en el caso del trabajo, el mayor acervo de capital interno y externo incrementa la demanda de trabajo al incrementar el rendimiento de este factor, ${ }^{25}$ con una contribución estimada de 0.14 y $0.13 \%$ respectivamente; de igual manera que un incremento de los precios de los bienes de inversión y del tipo de cambio real de $1 \%$, incidirían positivamente en la demanda de trabajo en 0.39 y $0.04 \%$ respectivamente.

Mientras que la demanda de capital interno aumenta a partir de la estructura planteada con el uso de los factores capital externo y el trabajo 0.09 y 0.9 respectivamente, destacando que este último factor aumenta la demanda de capital interno dada su complementariedad. Por otro lado, al incrementarse los precios de los bienes de inversión y de una depreciación cambiaria esta demanda se vería reducida en 0.8 y $0.2 \%$ respectivamente.

Finalmente, la demanda de capital externo, se verá incrementada por una mayor utilización tanto del capital interno como del trabajo, aunque cabe resaltar que esta aumenta más que proporcionalmente con el trabajo en casi 2.5 y se vería disminuida por el efecto de una depreciación cambiaria real $0.4 \%$.

Cabe señalar que en caso de aumentos en los precios de los bienes de capital y/o depreciaciones cambiarias reales disminuirá la demanda de capital interno y este a su vez reduciría la mano de obra.

Esto implica que una expansión en el factor trabajo se vería reflejado en un aumento mas que proporcional en los bienes de importación que de exportación, con un probable efecto adverso en la balanza comercial. En otras palabras, una apreciación cambiaria real, reduciría la tasa de retorno del capital e incrementaría la tasa de retorno del trabajo.

Así mismo, un aumento de la tasa de retorno del capital, tendería a atraer la inversión extranjera. Si a consecuencia de una devaluación o depreciación del peso mexicano, asumiendo por ejemplo que los precios internos de la producción permanecieran sin cambio, las estimaciones indican que el resultado neto sería una caída en la tasa de retorno del trabajo y un incremento en la tasa de retorno del capital.

Finalmente, podemos identificar los efectos distributivos de cambios en los precios de las variables de cantidades. En los resultados anteriores se indicó que un depreciación favorecería al capital sobre el trabajo, pero que una apreciación tendría un efecto opuesto.

24 El resultado que un aumento en la dotación del factor doméstico, puede conducir a una contracción en la producción de algún tipo de bien, es bien conocido como el que se presenta en el modelo de 2 factores productivos y 2 tipos de bienes son producidos (Teorema SamuelsonRybzcynski), aunque este resultado no necesariamente se mantiene, si el número de factores y la producción es incrementado o si se permite un proceso de producción conjunta.

25 En el apéndice $\mathrm{C}$ se presenta una prueba de Wald, de no linealidad de coeficientes. 
Similarmente, podemos encontrar que un aumento en el precio de los bienes de consumo desincentivaría la demanda de trabajo, a fin de sustituirlo por capital, que se usa en menor intensidad; al igual que un incremento en el precio de los bienes de inversión reduciría la demanda de capital interno, pero favorecería al capital externo.

Recordando que la oferta de trabajo y capital son exógenos, podemos deducir, que un aumento en el precio de los bienes de consumo, de inversión y comerciables internacionalmente, expandiría el producto total de la economía y la participación del trabajo por medio de una utilización mas intensiva de este factor; en tanto que una depreciación cambiaria real incrementaría el producto global de la economía, mayormente a través de las exportaciones y un aumento de los precios de los bienes de capital incrementaría una mayor demanda neta de bienes de capital externo, lo que en caso de traducirse en una mayor dotación de este factor incrementaría el producto global de la economía.

\section{Conclusiones}

Es importante destacar que para el caso de México no es aplicable la teoría del crecimiento endógeno al menos en el corto plazo, ya que los resultados apuntan a la importancia que tienen otros factores como precios relativos y dotación de factores en donde la política económica juega un papel preponderante

El enfoque utilizado en este documento para estimar las ecuaciones de importaciones y exportaciones parece tener algunas ventajas sobre el enfoque tradicional; ya que la demanda de importaciones reportada aquí es mas general y no se soporta con el supuesto de que todos los factores domésticos así como los componentes del producto global tienen que ser agregados.

Adicionalmente, para economías pequeñas es mucho mas útil analizar las exportaciones como determinadas primeramente por las condiciones de oferta más que por condiciones de demanda externa. Esto no quiere decir que variables como el producto o el ingreso externo o del resto del mundo no sean variables importantes en determinar las exportaciones de un país pequeño; ${ }^{26}$ ya que un cambio en el producto externo puede cambiar las curvas de oferta y demanda mundiales de un bien específico, e inducir a un cambio en su precio; pero el país pequeño es incapaz de afectar la curva de oferta mundial y por consecuencia pude ofrecer cualquier cantidad que se desee a los precios mundiales corrientes.

Este enfoque permite la estimación simultánea de las funciones de importaciones y exportaciones, así como la oferta y las funciones inversas de demanda de otros bienes y factores internos respectivamente. Este procedimiento no solo asegura que las ecuaciones de comercio exterior sean consistentes con la tecnología subyacente, pero también con el uso de supuestos de la tecnología sobre la demanda derivada y los sistemas de oferta y luego entonces algún conocimiento o luz sobre las posibilidades de sustitución existentes en la tecnología.

Es desde luego deseable, desagregar los factores productivos y la producción de los diversos tipos de bienes aún mas, sin embargo los trabajos que siguen

26 Un trabajo importante sobre el tema fué llevado a cabo por J. Salas Martín del Campo y J. J. Sidaoui Dib (1983). 
esta tendencia muestran que en la medida en que aumentan las ecuaciones, la estimación simultanea se vuelve mas difícil, incrementando significativamente los costos computacionales y alguna de las condiciones de regularidad son rápidamente violadas. La dirección natural para investigaciones futuras parece apuntar hacia una mayor desagregación de factores productivos y tipo de bienes producidos para implementar una función de producción agregada con un sistema de (costo - beneficio) que añada funciones; lo cual conducirá a sistemas de bloques recursivos simultáneos, los cuales serían estimados bajo condicionantes de Step-Wise. Este enfoque, sin embargo, necesariamente asumiría que los factores productivos y la producción de los diferentes tipos de bienes pueden en verdad ser desagregados en un número de componentes mayores.

\section{Apéndice A.}

Como se mencionó el Método Generalizado de Momentos, es un estimador robusto que 110 requiere información exacta de la distribución de los errores. No obstante, a fin de probar la no linealidad en los parámetros, se estimaron, estimaciones simultaneas del sistema por medio de Mínimos Cuadrados en Tres Etapas.

Los resultados son muy similares a los reportados por el Método Generalizado de Momentos, tanto a nivel de coeficientes individuales como los signos esperados, la significancia estadística, así como las pruebas generales coeficientes de determinación, suma cuadrada de residuales y las pruebas de D.W.

$$
\begin{aligned}
& D D L_{y}=0.005+0.343^{*} D D L B^{C}+0.121^{*} D D L B^{l} \\
& \text { (1.08) }(6.36)^{*} \quad(5.20)^{*} \\
& +0.018^{*} D D L B^{X} \quad-0.021^{*} D D L B^{M} \\
& \text { (1.19) } \quad(-1.00) \\
& +0.000006^{*} T R E N D+0.275^{*} D D L y_{(-1)} \\
& \text { (0.12) } \\
& (5.86)^{*}
\end{aligned}
$$

$\begin{array}{llll}\text { R-squared } & 0.945937 & \text { Mean dependent var } & 0.026996 \\ \text { Adjusted R-squared } & 0.942847 & \text { S.D. dependent var } & 0.044945 \\ \text { S.E. of regression } & 0.010745 & \text { Sum squared resid } & 0.012122 \\ \text { Durbin-Watson stat } & \mathbf{1 . 8 0 7 2 6 4} & & \end{array}$

$$
\begin{aligned}
& D D L B^{C}=0.161^{*} D D L H^{H T}+0.112^{*} D D L K^{E}+0.094^{*} D D L P^{C} \\
& \begin{array}{lll}
(2.69)^{*} & (5.83)^{*} & (0.76)
\end{array} \\
& -0.0003^{*} D D L P^{l} \quad-0.012^{*} D D L T C^{R} \\
& (-0.003) \quad(-0.43)
\end{aligned}
$$




$$
\begin{gathered}
+0.211^{*} D D L B_{(-1)}^{C} \\
(3.70)^{*}
\end{gathered}
$$

$\begin{array}{llll}\text { R-squared } & 0.689072 & \text { Mean dependent var } & 0.030114 \\ \text { Adjusted R-squared } & 0.674266 & \text { S.D. dependent var } & 0.046331 \\ \text { S.E. of regression } & 0.026442 & \text { Sum squared resid } & 0.073416 \\ \text { Durbin-Watson stat } & 1.881264 & & \end{array}$

$$
\begin{aligned}
& D D L B^{l}=0.039+0.458^{*} D D L H^{H T}+0.241^{*} D D L K^{E} \\
& (6.91)^{*} \quad(5.20)^{*} \quad(10.89)^{*} \\
& +0.343^{*} D D L K^{l}-0.513^{*} D D L P^{C} \\
& (15.20)^{*} \quad(-3.97)^{*} \\
& +0.526^{*} D D L P^{l}-0.151^{*} D D L P^{B C} \\
& (3.35)^{*} \quad(-3.09)^{*} \\
& -0.154^{*} D D L B_{(-1)}^{l} \\
& (-6.41)^{*}
\end{aligned}
$$

R-squared

Adjusted R-squared

S.E. of regression

Durbin-Watson stat
0.986397

0.985339

0.020785

1.301037
Mean dependent var

S.D. dependent var

Sum squared resid

0.025024

0.171658

0.038881

$$
\begin{aligned}
& D D L K^{X}=0.268^{*} D D L H^{H T}+0.106^{*} D D L K^{E}-0.523^{*} D D L P^{C} \\
& \text { (1.37) }(2.43)^{*} \quad(-1.73)^{*} \\
& -0.943^{*} D D L P^{l}+0.612^{*} D D L T C^{R} \\
& (-2.78)^{*} \quad(8.87)^{*} \\
& +0.0000000 .2^{*} T R E N D \quad+0.490^{*} D D L B_{(-1)}^{X} \\
& (0.18) \quad(8.46)^{*}
\end{aligned}
$$

R-squared

Adjusted R-squared

S.E. of regression

Durbin-Watson stat
0.865292

0.857594

0.079721

1.970206
Mean dependent var

0.101830

S.D. dependent var

0.211255

Sum squared resid

0.667318 


$$
\begin{aligned}
& D D L B^{M}=1.342^{*} D D L H^{H T} \quad-0.464^{*} D D L K^{l}+0.048^{*} D D L P^{C} \\
& (5.63)^{*} \quad(-5.27)^{*} \quad(0.11) \\
& +0.278^{*} D D L P^{l}-0.183^{*} D D L T C^{R} \\
& (0.69) \quad(-1.75)^{*} \\
& +0.601^{*} D D L B_{(-1)}^{M} \\
& (8.87)^{*}
\end{aligned}
$$

R-squared

Adjusted R-squared

S.E. of regression

Durbin-Watson stat
0.570009

0.549136

0.100150

2.055552
Mean dependent var

0.087602

S.D. dependent var

0.149152

Sum squared resid

1.033100

$$
\begin{aligned}
& D D L H^{H T}=-0.046+0.142^{*} D D L K^{E}+0.170^{*} D D L K^{l} \\
& \begin{array}{lll}
(-12.37)^{*} & (8.32)^{*} \quad(8.85)^{*}
\end{array} \\
& -0.221^{*} D D L P^{C} \quad-0.398^{*} D D L P^{l} \\
& (-2.39)^{*} \quad(3.84)^{*} \\
& +0.074^{*} D D L T C^{R} \\
& (3.50)^{*}
\end{aligned}
$$

$\begin{array}{llll}\text { R-squared } & 0.333020 & \text { Mean dependent var } & -0.012096 \\ \text { Adjusted R-squared } & 0.825512 & \text { S.D. dependent var } & \mathbf{0 . 0 5 8 1 1 5} \\ \text { S.E. of regression } & 0.024275 & \text { Sum squared resid } & 1.063055 \\ \text { Durbin-Watson stat } & 1.914107 & & \end{array}$

\begin{tabular}{cccc}
\hline$D D L K^{l}$ & $=0.037$ & $+0.044^{*} D D L K^{E}$ & $+1.455^{*} D D L H^{H T}$ \\
& $(2.80)^{*}$ & $(0.87)$ & $(7.60)^{*}$ \\
$+0.893^{*} D D L P^{C}$ & $-1.072^{*} D D L P^{l}$ \\
& $(3.81)^{*}$ & $(-4.03)^{*}$ \\
$-0.208^{*} D D L T C^{R}$ & $+0.417^{*} D D L K_{(-1)}^{l}$ \\
$(-4.03)^{*}$ & $(9.83)^{*}$ \\
\hline
\end{tabular}

R-squared

Adjusted R-squared

S.E. of regression

Durbin-Watson stat
0.919240

0.914669

0.062523

1.985551
Mean dependent var

$-0.015390$

S.D. dependent var

Sum squared resid

0.214037

0.414374 


$$
\begin{array}{cccc}
D D L K^{E} & =0.137 & +0.200^{*} D D L K^{l} & +2.732^{*} D D L H^{H T} \\
(7.75)^{*} & (2.41)^{*} & (10.67)^{*} \\
-1.578^{*} D D L P^{C} & +1.333^{*} D D L P^{l} \\
(-3.38)^{*} & (2.62)^{*} \\
-0.345^{*} D D L T C^{R} & -0.215^{*} D D L K \\
(-4.70)^{*} & (-3.79)^{*}
\end{array}
$$

$\begin{array}{llll}\text { R-squared } & 0.902682 & \text { Mean dependent var } & -0.049870 \\ \text { Adjusted R-squared } & 0.896535 & \text { S.D. dependent var } & 0.250589 \\ \text { S.E. of regression } & 0.080604 & \text { Sum squared resid } & 0.414374 \\ \text { Durbin-Watson stat } & 1.399199 & & \end{array}$

La prueba "t" de Student aparece entre paréntesis. * Prueba a un extremo. Estadísticamente significativa al 5\%.

Cabe señalar que la covarianza del determinante residual, fue:

\section{\begin{tabular}{|l|l|}
\hline Determinant residual covariance & $7.78 \mathrm{E}-27$ \\
\hline
\end{tabular}}

\section{Apéndice B.}

Con el propósito de revisar si el sistema era lineal, el algoritmo equivalente es el del método propuesto por Zellner (1962) Seemingly Unrelated Regression Equations, por lo que también se estimó el sistema con este método, para examinar la validez empírica de la hipótesis alternativa, de linealidad, lo cual se corroboró con los resultados que se presentan a continuación.

$$
\begin{array}{ccccc}
D D L_{y} & =0.007 & +0.217^{*} D D L B^{C} & +0.062^{*} D D L B^{l} & +0.031^{*} D D L B^{X} \\
& (2.21)^{*} & (12.64)^{*} & (1.62) & (3.81)^{*} \\
& -0.007^{*} D D L B^{M} & +0.00001^{*} T R E N D & \left.+0.238^{*} D D L y_{(}-1\right) \\
(-0.74) & (0.35) & (5.91)^{*}
\end{array}
$$

R-squared

Adjusted R-squared

S.E. of regression

Durbin-Watson stat
0.939398

0.935935

0.011376

1.803750
Mean dependent var

S.D. dependent var

0.026996

Sum squared resid

0.044945

0.013589

\section{$D D L B^{C}=0.269^{*} D D L H^{H T} \quad+0.077^{*} D D L K^{E}+0.174^{*} D D L P^{C}$}
$(5.14)^{*}$
$(4.64)^{*}$
$(2.00)^{*}$ 


$$
\begin{aligned}
& -0.086^{*} D D L P^{l} \quad-0.034^{*} D D L T C^{R} \\
& (-0.95) \quad(-1.47) \\
& +0.282^{*} D D L B_{(-1)}^{C} \\
& (5.49)^{*}
\end{aligned}
$$

$\begin{array}{llll}\text { R-squared } & 0.694149 & \text { Mean dependent var } & 0.030114 \\ \text { Adjusted R-squared } & 0.679585 & \text { S.D. dependent var } & 0.046331 \\ \text { S.E. of regression } & 0.026226 & \text { Sum squared resid } & 0.072217 \\ \text { Durbin-Watson stat } & 1.910900 & & \end{array}$

\begin{tabular}{cccc}
\hline$D D L B^{l}$ & $=0.040$ & $+0.569^{*} D D L H^{H T}$ & $+0.261^{*} D D L K^{E}$ \\
& $(8.06)^{*}$ & $(6.86)^{*}$ & $(12.23)^{*}$ \\
& $+0.281^{*} D D L K^{l}$ & $-0.143^{*} D D L P^{C}$ & $+0.096^{*} D D L P^{l}$ \\
& $(12.86)^{*}$ & $(-1.39)$ & $(0.77)$ \\
& $-0.124^{*} D D L P^{B C}$ & $-0.085^{*} D D L B_{(-1)}^{l}$ \\
& $(-3.52)^{*}$ & $(-5.29)^{*}$ & \\
\hline
\end{tabular}

$\begin{array}{llll}\text { R-squared } & 0.988402 & \text { Mean dependent var } & 0.025024 \\ \text { Adjusted R-squared } & 0.987500 & \text { S.D. dependent var } & 0.171658 \\ \text { S.E. of regression } & 0.019192 & \text { Sum squared resid } & 0.033150 \\ \text { Durbin-Watson stat } & 1.551938 & & \end{array}$

$$
\begin{aligned}
& D D L B^{X}=0.114^{*} D D L H^{H T} \quad+0.097^{*} D D L K^{E} \quad-0.625^{*} D D L P^{C} \\
& (0.72) \quad(3.80)^{*} \quad(-1.89)^{*} \\
& -0.931^{*} D D L P^{l}+0.281^{*} D D L T C^{R} \\
& (-2.52)^{*} \quad(5.93)^{*} \\
& +0.00000002^{*} T R E N D+0.636^{*} D D L B_{(-1)}^{X} \\
& (2.87)^{*} \quad(12.49)^{*}
\end{aligned}
$$

R-squared

Adjusted R-squared

S.E. of regression

Durbin-Watson stat
0.825582

0.815615

0.090713

2.251634
Mean dependent var

0.101830

S.D. dependent var

0.211255

Sum squared resid

0.864031 


$$
\begin{array}{cccc}
D D L B^{M} & =0.460^{*} D D L H^{H T} & -0.083^{*} D D L K^{l} & +0.031^{*} D D L P^{C} \\
(2.61)^{*} & (-2.23)^{*} & (0.87) \\
& & & \\
& 0.514^{*} D D L P^{l} & -0.225^{*} D D L T C^{R} & +0.695^{*} D D L B_{(-1)}^{M} \\
(1.31) & (-0.52) & (12.94)^{*}
\end{array}
$$

R-squared

Adjusted R-squared

S.E. of regression

Durbin-Watson stat
0.579825

0.559428

0.099001

2.366972
Mean dependent var

S.D. dependent var

Sum squared resid

0.087602

0.149152

1.009516

$$
\begin{array}{cccc}
D D L H^{H T} & =-0.056 & +0.122^{*} D D L K^{E} & +0.242^{*} D D L K^{l} \\
(-18.69)^{*} & (11.55)^{*} & (61.55)^{*} \\
& & & \\
-0.254^{*} D D L P^{C} & +0.482^{*} D D L P^{l} & +0.103^{*} D D L T C^{R} \\
(-2.88)^{*} & (4.91)^{*} & (5.78)^{*}
\end{array}
$$

R-squared

Adjusted R-squared

S.E. of regression

Durbin-Watson stat
0.780327 Mean dependent var

0.770157 S.D. dependent var

0.027803 Sum squared resid

1.559221
$-0.012468$

0.057993

0.083485

$$
\begin{array}{cccc}
D D L K^{l} & =0.228 & +0.496^{*} D D L K^{E} & +4.076^{*} D D L H^{H T} \\
(18.46)^{*} & (11.66)^{*} & (97.41)^{*} \\
+ & 1.044^{*} D D L P^{C} & -1.970^{*} D D L P^{l} & -0.424^{*} D D L T C^{R} \\
(2.91)^{*} & (-4.94)^{*} & (-5.86)^{*} \\
+ & 0.0112^{*} D D L K_{(-1)}^{l} & & \\
& (0.99)
\end{array}
$$

R-squared

Adjusted R-squared

S.E. of regression

Durbin-Watson stat
0.729114 Mean dependent var

0.713781 S.D. dependent var

0.114509

1.559519
0.015390

0.214037

Sum squared resid $\quad 1.389896$ 


\begin{tabular}{cccc}
\hline$D D L K^{E}$ & $=0.305$ & $+1.022^{*} D D L K^{l}$ & $+5.708^{*} D D L H^{H T}$ \\
& $(16.20)^{*}$ & $(14.19)^{*}$ & $(23.37)^{*}$ \\
$-0.724^{*} D D L P^{C}$ & $+1.849^{*} D D L P^{l}$ & $-0.652^{*} D D L T C^{R}$ \\
& $(-1.37)$ & $(3.14)^{*}$ & $(-6.71)^{*}$ \\
$-0.040^{*} D D L K_{(-1)}^{E}$ & & \\
$(-1.33)^{*}$ & \\
\hline
\end{tabular}

R-squared 0.661201 Mean dependent var 0.0498670

Adjusted R-squared

0.639803 S.D. dependent var 0.250589

S.E. of regression 0.150394 Sum squared resid

2.148754

Durbin-Watson stat

1.602860

La prueba "t" de Student aparece entre paréntesis. * Prueba a un extremo. Estadísticamente significativa al $5 \%$.

Cabe señalar que:

\section{\begin{tabular}{|l|l|}
\hline Determinant residual covariance & $3.59 \mathrm{E}-26$
\end{tabular}}

\section{Apéndice C.}

A continuación se presentan algunas pruebas de restricciones de coeficientes de Wald. Considerando un modelo de regresión general no-lineal. Tal que:

$$
y=x(\beta)+\varepsilon,
$$

donde $\beta$ es un vector de parámetros $k$ a estimar. Cualquier restricción en los parámetros puede escribirse:

$$
H_{0}=g(\beta)=0,
$$

donde $g$ es un vector de dimensión $q$ que son las restricciones impuestas sobre $\beta$. De tal manera que el estadístico de Wald es:

$$
W=n g(b)^{\prime}\left((\delta g / \delta \beta) V\left(\delta g / \delta \beta^{\prime}\right)\right)^{-1} g(b),
$$

siendo $n$ el número de observaciones y $b$ son los estimadores de los parámetros no restringidos; de esta forma $V$ es la varianza estimada que esta dada por:

$$
V=n s^{2}\left((\delta x / \delta \beta)\left(\delta x / \delta \beta^{\prime}\right)\right)^{-1}, \quad s^{2}=\left(\mu^{\prime} \mu / n-k\right) .
$$

Este estadistico tiene una distribución asintótica de una $\chi^{2}(q)$ donde $q$ es el número de restricciones bajo la hipótesis nula. 


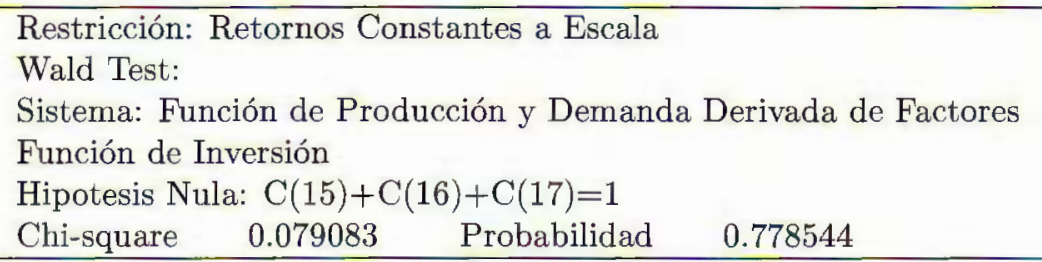

Como se puede apreciar, en la especificación de la función de producción de bienes de inversión se presentan rendimientos constantes a escala.

Restricción No-Lineal
Wald Test:
Sistema: Función de Producción y Demanda Derivada de Factores
Función de Demanda de Capital Interno y Externo
Hipotesis Nula: $\mathrm{C}(50)=\mathrm{C}(43) / .35$

Chi-squar $\quad 0.667534 \quad$ Probabilidad 0.413913

En las especificaciones de demanda de factores -capital interno y externo- se comprobó la hipótesis de no-linealidad de coeficientes del trabajo.

\section{Bibliografía}

Barro, R. y M. Sala I. (1995). Teoría del Crecimiento, McGraw Hill.

Berndt, E., B. Hall, R. Hall, and J. Hausman (1974). Estimation and Inference in Nonlinear Structural Models. Annals of Economic and Social Mesurement, pp. 653-65.

Berndt, E. and N. Savin (1975). Estimation and Hypotesis Testing in Singular Equation Systems with Autoregressive Disturbance. Econometrica, 43, pp. 937-57.

Berndt, E. (1991). The Practice of Econometrics Classic and Contemporary. Adison-Wesley. Christiansen, L., D. Jorgenson, and L. Lau (1973). Transcendental Logarithmic Production Frontiers. Review of Economics and Statistics, 55, pp. 28-45.

Diewert, W. (1973). Functional Forms for Profit and Transformation Functions. Journal of Economic Theory, 6, pp. 284-316.

Diewert, W. (1974). Applications of Duality Theory. In M.D. Intriligator and D.A. Kendrick (eds.). Frontiers of Quantitative Economics, Vol. 2, Amesterdam-North Holland.

Kohli, U. (1980). Non-Jointness and Factor Intensity in US Production. Manuscript.

Kohli, U. (1975). Canadian Technology and Derived Import Demand and Export Supply Functions. Ph.D. Thesis, University of British Columbia.

Leamer, E. and R. Stern (1970). Quantitative International Economics. Boston: Allyn and Bacon.

McFadden, D. (1978). The General Linear Profit Function, Chapter 12. In Melvyn Fuss and Daniel McFadden (eds.). Production Economics: A Dual Approach to Theory and Applications, Vol. 1, Amsterdam: North Holland,1, pp 269-286.

Salas Martín del Campo, J. y J. Sidaoui Dib (1983). Evolución y perspectivas de las exportaciones de manufactiuras. El Trimestre Económico, 50(4), Octubre-Diciembre, pp. $339-371$.

Tapia Maruri, J. y J. Cervantes González (1992). Un Modelo Econométrico del Impacto de la Apertura Comercial en la Balanza Comercial, la Actividad Económica y los Precios. En México hacia la Globalización. Editorial Diana, pags. 49-74. 
284 J. Tapia Maruri / Una función de producción agregada, su descomposición sectorial...

Uzawa, H. (1964). Duality Principles in the Theory of Cost and Production. International Economic Review, 5(2), pp. 216-220.

Zellner A., J. Kmenta; and J. Dréze (1966). Specification and Estimation of Cobb-Douglas Production Function Models. Econometrica, 34(3) October, pp. 784-795. 